\title{
Comment on Gu et al. entitled "Prognostic significance of HER2 expression based on trastuzumab for gastric cancer (ToGA) criteria in gastric cancer: an updated meta-analysis"
}

\author{
Jun Li • Juehua Jing
}

Received: 28 February 2014 / Accepted: 18 March 2014 /Published online: 25 March 2014

(C) International Society of Oncology and BioMarkers (ISOBM) 2014

Dear Editor,

We read with great interest the recent paper entitled "Prognostic significance of HER2 expression based on trastuzumab for gastric cancer (ToGA) criteria in gastric cancer: an updated meta-analysis" published online in Tumor Biology by $\mathrm{Gu}$ et al. [1]. We believe this is a well-conducted meta-analysis which enrolled only studies defining HER2 status by trastuzumab for gastric cancer (ToGA) criteria. However, the question being asked is an important one; there are some worthwhile issues we would like to communicate with the authors.

1. The authors searched only the following databases for studies: EMBASE (Jan 2008 to Nov 2013), Medline (Jan 2008 to Nov 2013), PubMed (Jan 2008 to Nov 2013), and Scopus (Jan 2008 to Nov 2013). The small number of required papers would be an important limitation of the review. We hope that more electronic databases can be systematically searched. Meanwhile, the authors did not describe the search strategy report for databases in detail, which plays an important role in systematic reviews. Manual searches were not expressed clearly. The lack of a manual search protocol may be regarded as a drawback of this meta-analysis. We suggest that the authors provide a complete search protocol.

2. Publication language in this meta-analysis was limited to English, so language bias may be unavoidable. Although the authors had mentioned it in the discussion, we suggest that there be no language limitation for the included studies to reduce the bias.

J. Li $(\bowtie) \cdot$ J. Jing

Department of Orthopaedics, The Second Hospital of Anhui Medical

University, Hefei, Anhui Province 230601, People's Republic of

China

e-mail: junliarmy@163.com
3. "Articles with insufficient published data in a full-text paper for determining an estimate of hazard ratio and $95 \%$ confidence interval were excluded," this exclusion criteria was not enough in the meta-analysis. We suggest that strict exclusion criteria should be described in detail.

4. The publication bias in this meta-analysis was assessed by visual examination of the funnel plot. However, the number of studies was less than ten; as far as we know, a funnel plot should be inspected visually to assess for publication bias, in meta-analyses with at least ten studies. Therefore, it is inappropriate.

5. The authors used an inverse variance (IV) random-effects model to pool the data in evaluating the association between HER2 expression and overall survival of gastric cancer based on six multivariate studies. In our opinion, the studies should be combined by DerSimonian and Laird random-effects model [2], which considers both within- and between-study variations.

In conclusion, we agree with the results of this metaanalysis. HER2 expression based on ToGA criteria is related to clinicopathological features of gastric cancer but not associated with the survival of the patients. To reach a definitive conclusion, more high-quality studies with larger sample sizes are still needed.

Conflicts of interest None.

\section{References}

1. Gu J, Zheng L, Wang Y, Zhu M, Wang Q, Li X. Prognostic significance of HER2 expression based on trastuzumab for gastric cancer (ToGA) criteria in gastric cancer: an updated meta-analysis. Tumour Biol. 2014. doi:10.1007/s13277-014-1693-7.

2. DerSimonian R, Laird N. Meta-analysis in clinical trials. Control Clin Trials. 1986;7(3):177-88. 